\title{
Efeito de duas intervencões visando o aumento do nível de átividade física de idosos inativos no tempo de lazer
}

\section{Effects of two interventions aiming at increasing the level of physical activity of physically inactive older adults in their leisure time}

\author{
Mirella Pinto Valerio ${ }^{1}$ \\ Francini Vilela Novais ${ }^{2}$ \\ Luiz Roberto Ramos²
}

\section{RESUMO}

Este estudo objetivou analisar o efeito de duas intervenções visando o aumento do nível de atividade física de idosos inativos no tempo de lazer (AFL) em idosos fisicamente inativos pertencentes a uma coorte urbana no sudeste do Brasil. Trata-se de um estudo experimental com 121 idosos de ambos os sexos de 60 a 89 anos de idade, residentes em um bairro de classe média da cidade de São Paulo alocados aleatoriamente em três grupos: 1) GS, grupo com sessões de exercícios físicos, ministradas duas vezes por semana, com duração de 60 minutos cada $(n=27), 2)$ GA, grupo com aconselhamento oral e por escrito $(n=22)$ e 3) GC, grupo controle, com uma recomendação por escrito $(n=72)$. As intervenções nos grupos GS e GA duraram três meses, com avaliações do nível de AFL realizadas no início, ao final da intervenção e três meses após sua conclusão. O desfecho "nível de AFL" foi mensurado pelo Questionário Internacional de Atividade Física (IPAQ), versão longa. Para comparações entre os grupos e momentos utilizou-se ANOVA com Post-hoc de Duncan. Houve um aumento significativo no tempo médio de prática de AFL ao final das intervenções, passando de zero para $111,1( \pm 5,3)$ minutos por semana no GS e $42,7( \pm 13,4)$ minutos por semana no GA. Após três meses do final das intervenções, o GS reduziu sua média de minutos por semana de AFL para $71,5( \pm 18,5)$ enquanto o GA aumentou para $71,8( \pm 18,3)$. Conclui-se que ambas intervenções foram efetivas para aumentar o nível de AFL de idoso inativos.

\section{PALAVRAS-CHAVE}

Envelhecimento; Atividade motora; Estilo de vida sedentário; Estudos de intervenção.

\begin{abstract}
This study aimed at analyzing the effect of two kinds of interventions in order to increase the level of leisure-time physical activities (LTPA) of physically inactive older adults who belong to an urban cohort in the southeast of Brazil. This experimental study was carried out with 121 older male and female adults between 60 and 89 years old who live in a middle-class suburb in São Paulo, SP. They were randomly divided into three groups: 1) GS, group with sixty-minute physical exercise sessions twice a week (n=27); 2) GA, group which got verbal guidelines and leaflets (n=22); and 3) GC, control group which got general written recommendations $(n=72)$. Interventions on groups $G S$ and GA lasted three months. Then, there was a three-month interval. LTPA levels were evaluated before the intervention, at its end and six months after its beginning. Results of the LTPA level were measured by the long version of the International Physical Activity Questionnaire (IPAQ). ANOVA and post-hoc Duncan were used to compare groups and periods. After the three-month intervention, GS increased from zero-minute LTPA per week to 111.1 ( \pm 5.3$)$ minutes per week whereas GA increased from zero to $42.7( \pm 13.4)$ minutes per week. When the intervention was ended, GS decreased the average to $71.5( \pm 18.5)$ minutes per week whereas GS increased to 71.8 $( \pm 18.3)$ minutes per week. Therefore, both interventions were effective to increase the level of inactive older adults' leisure-time physical activity.
\end{abstract}

\section{KEYWORDS}

Old age; Motor activity; Sedentary lifestyle; Studies of intervention.
Rev Bras Ativ Fis Saúde p. 765-773 DOI

http://dx.doi.org/10.12820/rbafs.v.19n6p765

1 Universidade Federal do Rio Grande. Instituto de Educação. Rio Grande (RS), Brasil.

2 Universidade Federal de São Paulo.

Departamento de Medicina Preventiva. São Paulo (SP), Brasil. 


\section{INTRODUCCÃO}

A população brasileira vem experimentando um envelhecimento populacional de maneira exponencial desde o início da década de 1960, quando a queda das taxas de fecundidade começou a alterar sua estrutura etária, estreitando progressivamente a base da pirâmide populacional ${ }^{1}$. Em 2010, o Censo Demográfico Nacional indicou que o número de pessoas com 60 anos ou mais superava 20 milhões².

O processo de envelhecimento é associado a alterações físicas, fisiológicas, psicológicas e sociais ${ }^{3}$. Algumas modificações no organismo como a redução da resistência física, da força muscular e da capacidade funcional geral são comuns entre os idosos, deixando-os mais propensos a desenvolverem enfermidades e influenciando negativamente em suas atividades cotidianas ${ }^{3}$.

A prática regular de atividade física é um dos meios mais eficazes para ajudar a prevenir doenças crônicas, promover a independência e aumentar a qualidade de vida na velhice. Manter tal hábito está associado à melhora da saúde, com a redução da morbidade e da mortalidade além do mesmo proporcionar melhoras nos aspectos psicológicos e sociais das pessoas que executam regularmente a referida atividade ${ }^{4}$.Embora seja recomendado que os idosos mantenham um estilo de vida ativo minimizando os riscos de doenças crônico-degenerativas ${ }^{3-6}$, eles ainda pertencem ao grupo etário com menor nível de atividade física $(\mathrm{AF})$, sendo que a frequência de sua prática tende a diminuir conforme aumenta a idade ${ }^{7-9}$.

Levantamento realizado pelo Ministério da Saúde, da Secretaria de Vigilância em Saúde e da Secretaria de Gestão Estratégica e Participativa, por meio do sistema VIGITEL (Vigilância de Fatores de Risco e Proteção para Doenças Crônicas por Inquérito Telefônico), demonstrou que a frequência de adultos que praticam atividade física no tempo livre é modesta em todas as cidades estudadas, como em São Paulo que atingiu apenas 28\%. Entre as pessoas com idade igual ou superior a 65 anos a prevalência de atividade física no tempo livre foi de 27,5 para homens e 18,9\% para mulheres ${ }^{7}$.

De acordo com o Centers for Disease Control and Prevention, a opção por uma vida mais ativa e saudável depende da oferta, da oportunidade de acesso a espaços favoráveis, da prática do lazer e/ou exercício físico, da complexidade da adesão e da continuidade à prática da atividade física ${ }^{10}$. Neste sentido, torna-se urgente investigar estratégias de promoção da AF em idosos insuficientemente ativos.

Estratégias fortemente recomendadas para o combate à inatividade física de idosos incluem abordagens como oferta de atividades físicas adaptadas, entregas rotineiras de mensagens curtas, orientação ou aconselhamento individual ou em grupo e incentivo a prática de atividade físicas ${ }^{10-13}$. Embora a literatura demonstre vários modelos de programas e intervenções voltadas para o aumento da prática de atividades físicas, não existe consenso sobre qual a melhor estrátegia para incrementar o nivel de atividade física no momento de lazer de idosos fisicamente inativos ${ }^{10-13}$. Nesse sentido, o presente estudo teve como objetivo analisar os efeitos de duas diferentes intervenções no nível de atividade física de idosos inativos no tempo de lazer. 


\section{MÉTODOS}

Trata-se de um estudo experimental, em que foram desenvolvidas duas intervenções voltadas à promoção de $\mathrm{AF}$ em idosos inativos de uma subamostra de Projeto EPIDOSO, envolvendo idosos residentes no bairro Vila Clementino, na cidade de São Paulo ${ }^{14}$.

O estudo foi aprovado pelo Comitê de Ética em Pesquisa da Universidade Federal de São Paulo, sob o número 0511/08.

\section{Recrutamento e Seleção}

Primeiramente, foi feita uma triagem em todos os idosos da coorte no período entre 2007-2010 (494 idosos) avaliados quanto ao nível de atividade física no tempo de lazer, por meio da aplicação do Questionário Internacional de Atividade Física (IPAQ - International Physical Activity Questionnaire) versão 8, forma longa e da Avaliação Geriátrica Ampliada (AGA).

Foram considerados elegíveis para este estudo 280 idosos que não atingiam o tempo de dez minutos semanais em atividades físicas no tempo de lazer (inativos) e que não possuíam impedimentos para a prática de atividades físicas moderadas. Estes voluntários foram convidados a participar do estudo e aleatoriamente alocados em dois grupos: intervenção $(n=159)$ e controle (121). Os sujeitos selecionados para intervenção foram subdivididos em outros dois grupos: Grupo sessões de exercício (GS) e Grupo aconselhamento (GA). Todos os convidados para participar do estudo, receberam informações sobre os objetivos e metodologia da pesquisa, seguindo de uma intervenção mínima que constava de uma breve recomendação por escrito para que acumulassem no mínimo 150 minutos de atividades físicas por semana ${ }^{4}$.

No total, 49 sujeitos selecionados para os grupos de intervenção aceitaram participar do estudo, assistiram a uma palestra feita por um geriatra do EPIDOSO abordando questões acerca do envelhecimento e atividade física e assinaram o termo de consentimento livre e esclarecido (TCLE). Os grupos ficaram assim constituídos (Figura 1).

\section{Intervenções}

Os integrantes do grupo sessão de exercícios (GS) participaram de aulas compostas de atividades aeróbicas e exercícios de flexibilidade, equilíbrio e fortalecimento muscular, duas vezes por semana, com duração de sessenta minutos cada, durante doze semanas. Todas as sessões foram realizadas no espaço cultural Pátio da Tribo da Universidade Federal de São Paulo.

Os integrantes do grupo aconselhamento (GA) participaram de uma única sessão de exercícios realizada coletivamente, na qual foram explicados e salientados os cuidados que deveriam ter quanto à postura, frequência, intensidade e duração dos exercícios que deveriam realizar. Receberam um folheto contendo benefícios e exemplos da prática regular de atividades físicas, e foram incentivados a praticá-la pelos próximos três meses. Durante este período, os idosos foram acompanhados mediante chamadas telefônicas realizadas após 45 e 90 dias do primeiro encontro, com durações aproximadas de dez minutos cada, quando eram aconselhados e estimulados à prática de atividades físicas. As informações obtidas nestes telefonemas, como tempo de AF, dificuldades encontradas e aconselhamentos realizados foram anotados em fichas individuais. 


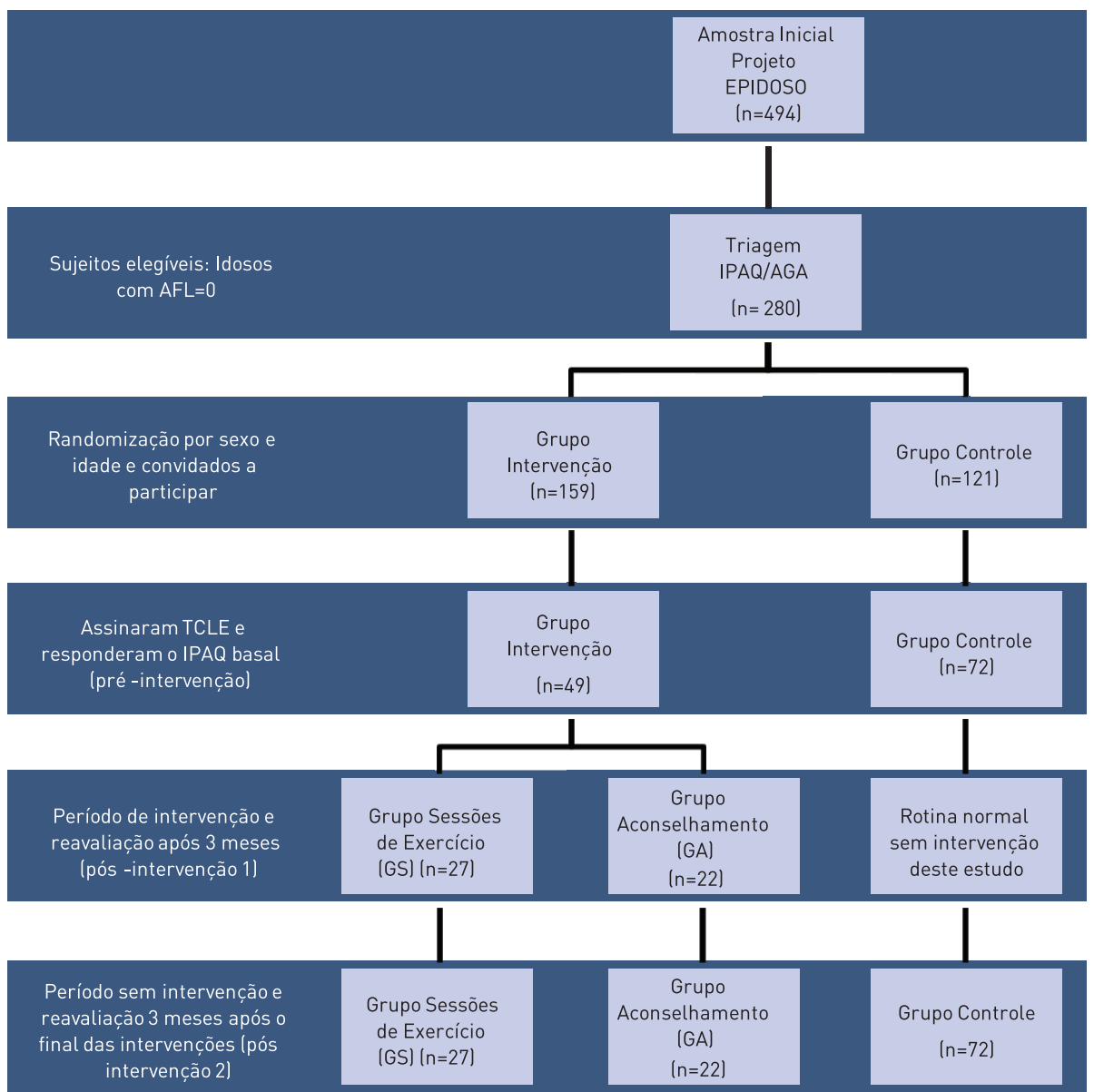

FIGURA 1 - Recrutamento e seleção dos participantes (n = Número de sujeitos; EPIDOSO = Epidemiologia do Idoso; $A F L$ = Atividade física no lazer; $I P A Q=$ Questionário Internacional de Atividade Física; AGA = Avaliação Geriátrica Ampliada).

Os indivíduos do grupo controle (GC) receberam apenas a intervenção mínima citada anteriormente seguiram sua rotina normal sem qualquer outra interferência deste estudo.

\section{Medidas}

- Nível de Atividade Física

O desfecho deste estudo "nível de atividade física no lazer" foi obtido por meio da aplicação do IPAQ, realizado em entrevista individual tendo como referência a última semana sendo consideradas as AFL feitas por mais de 10 minutos contínuos. O IPAQ é um instrumento específico que visa mensurar as atividades físicas realizadas no trabalho, no transporte, nas atividades domésticas e no lazer ${ }^{15} . \mathrm{Na}$ presente pesquisa, foi utilizado o domínio que avalia as atividades físicas de recreação, esporte ou lazer (usualmente identificada como atividades físicas de lazer).

Os participantes dos grupos de intervenção foram avaliados na linha de base (pré-intervenção), ao final de três meses de intervenção (pós-intervenção 1) e reavaliados três meses depois de cessada a intervenção (pós-intervenção 2). Os integrantes do grupo controle foram avaliados apenas nos momentos pré-intervenção e pós-intervenção 2 . O desfecho foi analisado tanto com relação ao percentual de mudança de comportamento no nível de AFL quanto nas diferenças de médias de minutos por semana nos diferentes tempos e grupos acima citados. 
Todas as aplicações do IPAQ foram feitas em forma de entrevista face a face por pesquisadores treinados para este fim e cegos com relação à alocação dos voluntários nos grupos.

- Variáveis demográficas, socioeconômicas e relativas à saúde As variáveis independentes incluídas no presente estudo foram: sexo (feminino/masculino), faixa etária em anos (de 60 a 65, de 66 a 70, de 71 a 75, de 76 a 80 , de 81 a 85 , de 86 a 90), estado conjugal (solteiro, casado, separado, viúvo), escolaridade (em anos de estudo: sem nenhum estudo, igual ou menos a 3 anos, de 4 a 7 anos, igual ou superior a 8 anos), arranjo domiciliar (mora sozinho ou acompanhado), renda pessoal mensal em salários mínimos (sem renda, até 1 SM, de 2 a 3 SM, de 4 a 10 SM, mais de 10 SM), quantidade de medicamentos ingeridos (não utiliza, de 1 a 2 , de 3 a 4 , de 5 a 6,7 ou mais) e doenças e agravos não transmissíveis (não apresenta, 1, de 2 a 4, igual ou superior a 5). Todos estes dados foram retiradas da Avaliação Geriátrica Ampla (AGA) proposta pela equipe de profissionais do EPIDOSO.

\section{Análise estatística}

Para a confecção do banco de dados, foi utilizado o programa Excel 2007 by Windows ${ }^{\circledR}$, através de dupla digitação dos referidos dados, posteriormente transferidos para o programa StatSoft ${ }^{\circledR}$ (Statistica analysis software system, version 7, 2004).

Para verificar se existia diferença proporcional de sexo e idades entre os grupos controle, aconselhamento e sessões de exercícios, empregou-se o teste de Qui-Quadrado, utilizando uma tabela de contingência, em um nível de significância de $5 \%$.

A fim de verificar se existiam diferenças significativas entre as variáveis independentes e as médias de tempo de atividade no momento pós-intervenção 2, foram realizadas análises de variância fatoriais dentro dos grupos GS, GA e GC também em um nível de significância de 5\%.

Com vistas à comparação das médias no tempo de AFL entre os diferentes grupos, nos momentos pós-intervenção 1 e pós-intervenção 2 , transformaramse as medidas originais através da função Logaritmo, para satisfazer os pré-requisitos do método de análise, e aplicou-se a Análise de Variância com Medidas Repetidas com um fator categórico de três níveis (Sessões, Aconselhamento e Controle). Posteriormente, aplicou-se o teste de Post-Hoc de Duncan, para determinar as diferenças, dois a dois, entre os níveis dos fatores estudados.

\section{RESULTADOS}

\section{Características gerais dos participantes}

Na presente pesquisa, dos 280 idosos elegíveis, 159 (56,8\%) deixaram de participar do processo ( 80 se recusaram a fazer parte do projeto e 79 estavam impossibilitados de cumprir as etapas do experimento por diversas razões). Não houve diferenças estatisticamente significativas entre as perdas e os que participaram do estudo em termos de idade e sexo ( $p>0,05$; dados não mostrados).

Os participantes tinham entre 60 e 89 anos, apresentando média de 71,5 anos (DP $=7,1)$ sendo a maior parte $(73,5 \%)$ do sexo feminino. Não houve diferença significativa entre os grupos em relação à distribuição por sexo $(\mathrm{p}=$ $0,29)$ e média de idade $(\mathrm{p}=0,66)$. 
A maior parte dos idosos era casada (52\%) e morava na companhia de outras pessoas $(85,1 \%)$. Com relação ao nível socioeconômico, a maioria recebia de três a dez salários mínimos mensais $(66,1 \%)$ e possuíam um tempo superior a oito anos de escolaridade $(69,4 \%)$. A presença de alguma doença crônica foi observada em $97,5 \%$ dos sujeitos e a maior parte deles $(90,9 \%)$ faz uso contínuo de ao menos um medicamento. $\mathrm{O}$ teste qui-quadrado não mostrou diferenças estatisticamente significativa entre os grupos para nenhuma das características acima relatadas.

Em relação à prática de AFL, no momento final das intervenções (pós-intervenção 1), a maioria dos idosos pertencente ao GS estava praticando algum tipo de atividade física por mais de 10 minutos (96,2\%), ao passo que no GA, apenas $36,3 \%$ dos casos. Na avaliação realizada três meses após o término das intervenções (pós-intervenção 2), constatou-se que no GS houve uma redução do percentual de idosos praticantes de $\operatorname{AFL}(55 \%)$ enquanto que no GA houve um aumento deste percentual (54,5\%). No GC foi observado um acréscimo de $23 \%$ de idosos que começaram a fazer algum tipo de AFL a ser considerada (Tabela 1).

TABELA 1 - Proporção de participantes em cada grupo que aderiram a algum tipo de atividade física no momento de lazer nos diferentes momentos avaliados

\begin{tabular}{lccc}
\hline Momento & GS \% (n) & GA \% (n) & GC \% (n) \\
\hline Pré-intervenção & $0(27)$ & $0(22)$ & $0(72)$ \\
\hline Pós-intervenção 1 & $96,29(26)$ & $36,36(08)$ & - \\
\hline Pós-intervenção 2 & $55,55(15)$ & $54,54(12)$ & $23,61(17)$ \\
\hline
\end{tabular}

GS = Grupo Sessão de Exercícios; GA = Grupo Aconselhamento; GC = Grupo Controle; \% = Frequência relativa; $\mathrm{n}$ = Frequência absoluta.

Na comparação entre as diferenças das médias de minutos por semana de AFL entre os grupos sessões de exercícios e aconselhamento, observou-se que no momento pós-intervenção 1 houve diferença significativa $(\mathrm{p}<0,006)$ entre eles. Entretanto, esta diferença não foi mantida no momento pós-intervenção 2 ( $\mathrm{p}=0,946)$.

No GS, houve uma redução significativa $(\mathrm{p}<0,001)$ entre a média de minutos de AFL no momento pós-intervenção 1 (111,1min/sem) e pós-intervenção 2 (71,5 min/sem). O mesmo não aconteceu com o GA em que as médias dos minutos semanais de atividades físicas aumentaram significativamente $(\mathrm{p}=0,045)$, passando de 42,7 min/sem para 71,8 min/sem no momento pós-intervenção 2 .

Analisando o comportamento do grupo controle no momento pós-intervenção 2, observou-se que seus integrantes apesar de aumentarem significativamente a média de minutos semanais de AFL (de zero para 43,3 $\mathrm{min} / \mathrm{sem}$ ) realizavam um tempo menor que os demais grupos $(\mathrm{p}=0,012)$. Alterações na atividade física ao longo do tempo são mostradas na Figura 2.

Não foi encontrada associação entre as variáveis independentes (idade, sexo, estado conjugal, escolaridade, moradia, renda, medicamentos e DCNT) com o aumento dos minutos de prática de $\mathrm{AF}$ dos integrantes dos três grupos da pesquisa no momento pós-intervenção 2 .

\section{DISCUSSÃO}

Os resultados deste estudo mostraram que após três meses da avaliação inicial, os grupos de intervenção tiveram um aumento significativo tanto na média de 


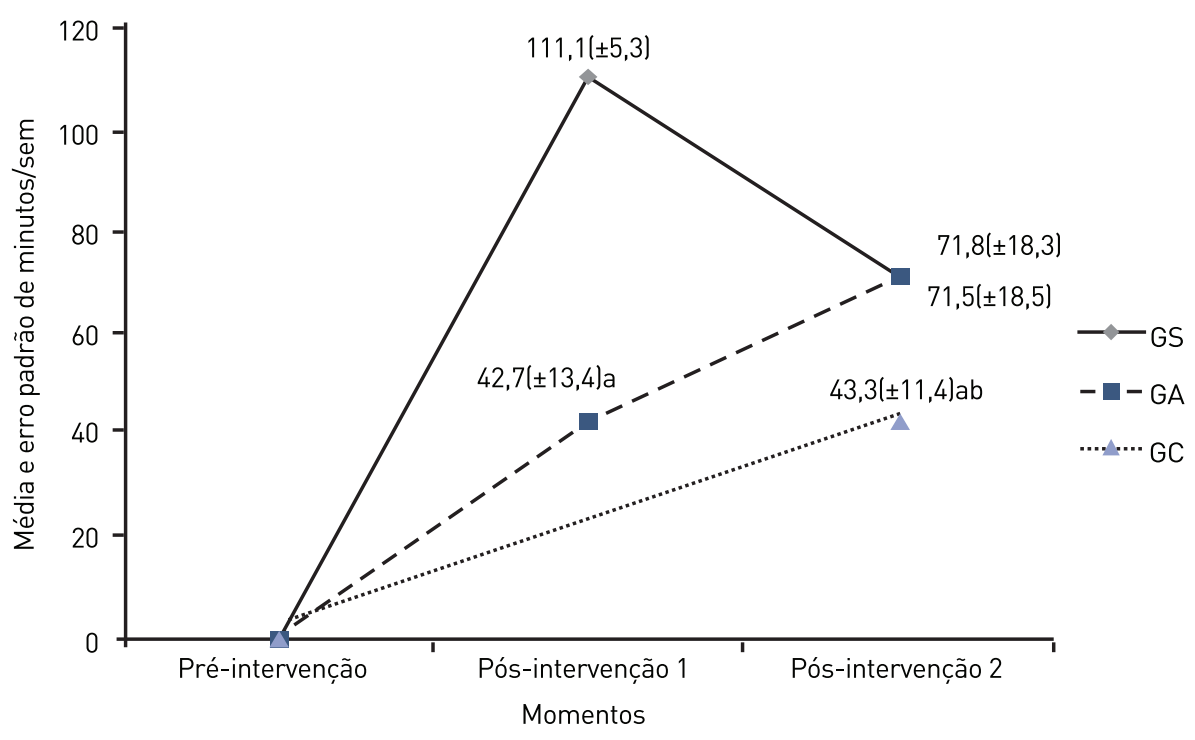

FIGURA 2 - Médias e erro padrão de minutos por semanas de AFL dos três grupos nos diferentes momentos (Valores expressos em minutos por semana; Valores de $p$ obtidos na ANOVA; post-hoc de Duncan: a $p<0,05$ versus GS; $b p<0,05$ versus $G A$.

minutos por semana quanto no nível de AFL, sendo mais expressivo no GS. $\mathrm{Na}$ avaliação seguinte (seis meses após a avaliação inicial), o percentual de indivíduos que praticavam algum tipo de AFL e a média de minutos por semana ficaram equiparados em ambos os grupos de intervenção sendo significativamente maior do que no GC.

Outros estudos também demonstraram que essas intervenções podem ser traduzidas em sucesso quanto à mudança de comportamento em idosos inati$\operatorname{vos}^{12-13,16-20}$. Em relação às intervenções realizadas em grupos com sessões de exercício, Van der Bij et al. (2002) $)^{12}$, em um estudo de revisão, constataram que esta estratégia é eficaz no aumento da participação dos idosos em atividade física encontrando uma taxa média entre os estudos de $84 \%$ ao final das intervenções em grupo. Cox et al. $(2003)^{16}$, referem que a prática da atividade física realizada em grupo e a curto prazo, alcança um maior engajamento dos participantes. A revisão sistemática realizada por Hong, Hughes e Prohaska $(2008)^{18}$, analisou estudos com idosos sedentários, entre os anos de 1980 e 2000 confirmou que, programas em grupo têm maior participação do que os realizados de forma individualizada.

$\mathrm{O}$ aumento no percentual e na média de minutos por semana de AFL do GA está de acordo com estudos que colocam o uso de materiais impressos ou telefonemas como uma excelente forma de motivar e auxiliar na automonitoração de idosos que ingressam em programas de atividades físicas ${ }^{13,17}$. Do mesmo modo, Pinto et al..$^{21}$ e Kolt et al..$^{22}$, utilizaram aconselhamentos e chamadas telefônicas para incentivar idosos à prática de atividades físicas, e obtiveram, ao final de três meses, um acréscimo de minutos por semana de atividade física (57,6 e 48,9 $\mathrm{min} / \mathrm{sem}$ respectivamente).

Após três meses do final das intervenções, observou-se que a diferença das médias de minutos por semana utilizados na prática de AFL encontrada nos grupos de intervenção deixou de existir. Entre as razões que podem ter levado os idosos do GS a reduzirem o nível de atividade física, podem estar a falta de alguns fatores presentes na intervenção, como as amizades, o convívio em 
grupo, as aulas ministradas por professor de educação física, monitoração constante na execução dos movimentos, e ainda a oferta de práticas no grupo de mesma faixa etária ${ }^{23-24}$. Tais fatores são fundamentais para motivar os idosos a participarem de programas de atividades físicas e contribuem para que os mesmos se sintam seguros durante a realização de atividades físicas ${ }^{25-27}$.

$\mathrm{O}$ aumento do número de idosos que deixaram de ser inativos e da média de minutos de AFL observado no GA pode ser atribuído ao estímulo individual realizado durante a intervenção, que os levou a incorporarem valores, transformando-os em hábitos e em automotivação para a prática das atividades físicas ${ }^{13,24-25,28}$.

Quanto ao grupo controle, a mudança do nível de atividades físicas pode ser explicada pelo fato do projeto EPIDOSO, por questões éticas, ter em sua rotina uma intervenção mínima de atividade física aos seus participantes que envolve breve aconselhamento oral e por escrito ${ }^{29-30}$.

Uma das limitações do estudo está no fato do desfecho ser obtido por meio de questionário o que pode ocasionar um viés de recordação entre os idosos avaliados ou mesmo uma supervalorização do tempo real de atividade física, pois trata-se de um comportamento desejável por todos. A generalização destes achados se torna limitada tendo em vista que foi utilizada uma amostra de conveniência na qual os participantes do estudo residem em um bairro de classe média e possuem um bom nível de escolaridade e renda. Todos os participantes residiam na mesma região, o que pode ter influenciado vizinhos e até mesmo parentes que faziam parte do GC para iniciarem alguma atividade física.

Os resultados apresentados mostraram que enquanto foi ofertado um estímulo, a estratégia utilizada no GS foi mais efetiva no aumento da AFL quando comparada ao GA e quando este foi retirado, ambas as estratégias utilizadas apresentaram efeitos semelhantes no nível de atividade física no momento de lazer de indivíduos idosos inativos. Nesse contexto, as intervenções realizadas no GS e no GA tiveram uma importante contribuição tanto para o aumento do percentual de idosos praticantes de AFL como nas médias de minutos por semana.

\section{REFERÊNCIAS}

1. Ramos LR, Veras RP, Kalache A. Envelhecimento populacional: uma realidade brasileira. Rev Saude Publica. 1987; 21(3):211-24.

2. Brasil. Instituto Brasileiro de Geografia e Estatística. Sinopse do Censo Demográfico 2010. Rio de Janeiro: Ministério do Planejamento, Orçamento e Gestão. Instituto Brasileiro de Geografia e Estatística, 2011.

3. Hayflick L. Como e por que envelhecemos.Rio de Janeiro: Campus, 1996.

4. Nelson ME, Rejeski WJ, Blair SN, Duncan PW, Judge JO, King AC, et al., Physical activity and public health in older adults: recommendation from the American College of Sports Medicine and the American Heart Association. Circulation. 2007;116:1094-105.

5. United States Department of Health and Human Services. USDHHS - Physical Activity Guidelines Advisory Committee. (PAGAC) Washington, DC; 2008. Available from: http://www.health.gov/paguidelines.

6. Powell KE, Paluch AE, Blair SN. Physical activity for health: What kind? How much? How intense? On top of what? Ann Rev Public Health. 2011; 32:349-65.

7. Brasil. Ministério da Saúde. Secretaria de Vigilância em Saúde. Vigitel Brasil 2011: Vigilância de Fatores de Risco e Proteção para Doenças Crônicas por Inquérito Telefônico. Brasília: Ministério da Saúde, 2012.

8. Livramento GA, Fagundes PPAN, Winter GR, Bernardes VP, Krause MP. Estudo longitudinal do nível de atividade física de mulheres idosas. Rev Bras Ativ Fís Saúde. 2012;17(6):552-61.

9. Del Duca GF, Nahas MV, Garcia LMT, Mota J, Hallal PC, Peres MA. Prevalence and sociodemographic correlates of all domains of physical activity in Brazilian adults. Prev Med. 2013;56: 99-102. 
10. Centers for Disease Control and Prevention. Strategies to Prevent Obesity and Other Chronic Diseases: The CDC Guide to Strategies to Increase Physical Activity in the Community. Atlanta: U.S. Department of Health and Human Services; 2011.

11. Task Force on Community Preventive Services. Recommendations to increase physical activity in communities. Am J Prev Med. 2002;22(Suppl 4):S67-72.

12. Van der Bij AK, Laurant MG, Wensing M. Effectiveness of physical activity interventions for older adults: a review. Am J Prev Med. 2002;22(2):120-33.

13. Foster C, Hillsdon M, Thorogood M, Kaur A, Wedatilake T. Interventions for promoting physical activity. Cochrane Database Syst Rev. 2005; 25(1):1-92.

14. Ramos LR. Fatores determinantes do envelhecimento saudável em idosos residentes em centro urbano: Projeto EPIDOSO, São Paulo. Cad Saúde Pública. 2003; 19:793-98.

15. Craig CL, Marshall AL, Sjöström M, Bauman AE, Booth ML, Ainsworth BE, et al. International Physical Activity Questionnaire: 12-Country Reliability and Validity. Med Sci Sports Exerc. 2003; 35(8):1381-95.

16. Cox KL, Burke V, Gorely TJ, Beilin LJ, Puddey IB. Controlled Comparison of Retention and Adherence in Home- vs Center-Initiated Exercise Interventions in Women Ages 40-65 Years: The S.W.E.A.T. Study (Sedentary Women Exercise Adherence Trial). Prev Med. 2003;36(1):17-29.

17. Marshall AL, Owen N, Bauman AE. Mediated approaches for influencing physical activity: update of the evidence on mass media, print, telephone and website delivery of interventions. J Sci Med Sport. 2004;7(Suppl 1):S74-80.

18. Hong SY, Hughes S, Prohaska T. Factors affecting exercise attendance and completion in sedentary older adults: a meta-analytic approach. J Phys Act Health. 2008; 5(3):385-97.

19. Hughes SL, Seymour RB, Campbell RT, Whitelaw N, Bazzarre T. Best-Practice Physical Activity Programs for Older Adults: Findings from the National Impact Study. Am J Public Health.2009; 99(2):362-68.

20. Brassington GS, Atienza AA, Perczek RE, DiLorenzo TM, King AC. InterventionRelated Cognitive Versus Social Mediators of Exercise Adherence in the Elderly. Am J Prev Med. 2002;23(Suppl 2):S80-86.

21. Pinto BM, Lynn H, Marcus BH, DePue J, Goldstein MG. Physician-based activity counseling: intervention effects on mediators of motivational readiness for physical activity. Ann Behav Med. 2001; 23(1):2-10.

22. Kolt GS. Schofield GM, Kerse N, Garrett N, Oliver M. Effect of telephone counseling on physical activity for low-active older people in primary care: a randomized controlled trial. J Am Geriatr Soc. 2007; 55(7):986-92.

23. Shutzer KA, Graves S. Barriers and motivations to exercise in older adults. Prev Med. 2004;39:1056-61.

24. Ferreira LP, Najar AL. Programas e campanhas de promoção da atividade física. Ciênc Saúde Coletiva. 2005; 10: 207-19.

25. Brawley LR, Rejeski WJ, King AC. Promoting Physical Activity for Older Adults The Challenges for Changing Behavior. Am J Prev Med. 2003; 25(3Suppl 2):S172-183.

26. Freitas CMSM, Santiago MS, Viana AT, Leão AC, Freyre C. Aspectos motivacionais que influenciam a adesão e manutenção de idosos a programas de exercícios físicos. Rev Bras Cineantropom Desempenho Hum. 2007; 9(1): 92-100.

27. Nascimento CMC, Gobbi S, Hirayama MS, Brazão MC. Nível de atividade física e as principais barreiras percebidas por idosos de Rio Claro. Rev Educ Fis. 2008; 1(19): 109-18.

28. Jong J, Lemmink KA, Stevens M, Greef MH, Rispens P, King AC, MulderT. Six-month effects of the Groningen active living model (GALM) on physical activity, health and fitness outcomes in sedentary and underactive older adults aged 55-65. Patient Educ Couns. 2006; 62(1):132-41.

29. Calfas KJ, Long BJ, Sallis JF, Wooten WJ, Pratt M, Patrick K. A controlled trial of physician counseling to promote the adoption of physical activity. Prev Med. 1996:5, 225-33.

30. Armit CM, Brown WJ, Marshall AL, Ritchie CB, Trost SG, Green A, Bauman AE. Randomized trial of three strategies to promote physical activity in general practice. Prev Med. 2009; 48: 156-63.I 